Bull. Austral. Math. Soc.

13А99, 13G05, 46H40, 46J20, 46J45, 46J99

VOL. 60 (1999) [153-161]

\title{
TOPOLOGICALLY SIMPLE BANACH ALGEBRAS WITH DERIVATION
}

\author{
El Hossein Illoussamen and Volker Runde
}

It is not known if a commutative, topologically simple, radical Banach algebra exists. If, however, every derivation on such an algebra is continuous, this yields the automatic continuity of all derivations on commutative, semiprime Banach algebras. Utilising techniques used by Thomas in his proof of the Singer-Wermer conjecture, we show that, if $A$ is a commutative, topologically simple Banach algebra with a non-zero derivation on it, then a quotient of a certain localisation of $A$ has a power series structure. A pivotal rôle is played by what we call ample sets of denominators.

\section{INTRODUCTION}

In [10], Johnson proved that derivations on commutative, semisimple Banach algebras are automatically continuous and thus, by the classical Singer-Wermer theorem [12], equal to zero. This result confirmed a long open conjecture by Kaplansky. In [6], Dixon observed that the class of semiprime Banach algebras was a natural extension of the class of semisimple Banach algebras and asked if the automatic continuity results known to hold for semisimple Banach algebras extend to this larger class. The problem of whether every derivation on a (commutative) semiprime Banach algebra is automatically continuous has been open ever since.

Partial results - all positive - are given, for instance, in [8], [11], and [9]. All these results utilise the connections of the automatic continuity question for derivations on commutative, semiprime Banach algebras with the perhaps deepest open problem in general Banach algebra theory, the closed ideal problem, which were first discovered by Cusack [5].

Recall that a Banach algebra $A$ with $A^{2} \neq\{0\}$ is called topologically simple if it has no two-sided closed ideals other than $\{0\}$ and $A$. A topologically simple Banach algebra is necessarily primitive or radical. The closed ideal problem is the question of

Received 3rd February, 1999

The results presented in this note were obtained while the second author was visiting the E.N.S. Takaddoum. Financial support was provided by the Deutsche Forschungsgemeinschaft (DFG) and the Centre National de Coordination et de Planification de la Recherche Scientifique et Technique (CNR) of Morocco. This support and the warm hospitality of the functional analysis group at the E.N.S. Takaddoum are gratefully acknowledged by the second author.

Copyright Clearance Centre, Inc. Serial-fee code: 0004-9729/99 \$A2.00+0.00. 
whether there is a commutative, topologically simple Banach algebra other than $\mathbb{C}$. If a commutative, topologically simple Banach algebra other than $\mathbb{C}$ exists, it must be radical, and it is also not difficult to see that it must also be an integral domain. Let $A$ be a commutative Banach algebra which is an integral domain - by [8], the automatic continuity of derivations on commutative, semiprime Banach algebras follows once it has been established for integral domains, and let $I$ denote the intersection of the non-zero closed ideals of $A$. It is easy to see that $I$ is either $\{0\}$ or a topologically simple, radical Banach algebra. If $I=\{0\}$, that is, if $\{0\}$ is accessible in the terminology of [4], then every derivation on $A$ is continuous as a simple consequence of $[4,1.1 \mathrm{Lemma}]$. It follows that every derivation on $A$ is continuous if $\mathbb{C}$ is the only commutative, topologically simple Banach algebra. The results from $[\mathbf{8}, \mathbf{1 1}, \mathbf{9}]$ all give, in one way or another, a necessary condition for $I$ to be zero.

In this note we do not even attempt to make any step towards a resolution of the closed ideal problem. Instead, we attack the mythological beasts directly: We consider derivations $D: A \rightarrow A$, where $A \neq \mathbb{C}$ is a commutative, topologically simple Banach algebra. We are keenly aware of the fact that our approach might ultimately turn out to be a contribution to the theory of the empty set. However, since the closed ideal problem has been open since the early days of Banach algebra theory, and no solution even seems to be in sight, we feel justified to proceed this way in view of [11, Theorem 6$]$ : If all derivations on commutative, topologically simple Banach algebras are automatically continuous, then all derivations on commutative, semiprime Banach algebras are continuous. Certainly, the ideas contained in $[8,11,9]$ do not help us any further. Instead, we shall borrow heavily from the first part of Thomas' proof of the Singer-Wermer conjecture [15], and show that, under certain additional conditions on $D$, a certain quotient of a localisation of $A$ has a power series structure.

\section{LOCALISATIONS OF BANACH ALGEBRAS}

In [10], Johson reduced the Singer-Wermer conjecture to the case of commutative, local Banach algebras, that is, commutative radical Banach algebras with identities adjoined. The Singer-Wermer conjecture was thus equivalent to the question of whether a derivation on a commutative, local Banach algebra could attain invertible values. If $A$ is a commutative, local Banch algebra, $D: A \rightarrow A$ is a derivation, and $z \in \operatorname{rad}(A)$ is such that $D z \in \operatorname{Inv}(A)$, then $A \ni a \mapsto(D z)^{-1} D$ is a derivation attaining the value 1 at $z$. Under these hypotheses, Thomas showed in [15] that $A / \bigcap_{n=1}^{\infty} z^{n} A$ has a power series structure (and, secondly, that this is irreconcilable with $A$ being a commutative, local Banach algebra).

We wish to obtain a similar power series structure result to that in $[\mathbf{1 5}]$, when $A$ is a commutative, topologically simple Banach algebra, and $D: A \rightarrow A$ is a non-zero derivation. In this context, however, we have no invertible elements at our disposal. 
What we shall do therefore, is to make certain elements of $A$ artificially invertible by localising the algebra.

Let $A$ be a commutative algebra, and let $S$ be a multiplicative subsemigroup of $A$ containing no divisors of zero, that is, a set of denominators. Define an equivalence relation $\sim$ on $A \times S$ through

$$
(a, s) \sim\left(a^{\prime}, s^{\prime}\right) \quad: \Longleftrightarrow \quad a s^{\prime}=a^{\prime} s \quad\left(a, a^{\prime} \in A, s, s^{\prime} \in S\right) .
$$

and let $S^{-1} A$ denote the set of the corresponding equivalence classes; write $a / s$ or $a s^{-1}$ for the equivalence class of $(a, s) \in A \times S$. With the usual rules for calculating with fractions, $S^{-1} A$ becomes a commutative, unital algebra, in which every element of $S$ becomes invertible. It is called the localisation of $A$ with respect to $S$.

REMARKS 1. Unlike most standard texts on commutative algebra, such as [3], we do not assume $A$ to be unital. If $s \in S$ is an arbitrary element, the map $A \ni a \mapsto a s / s$ is an embedding which does not depend on the choice of $s$; we shall therefore simply view $A$ as a subalgebra of $S^{-1} A$. If $A$ is non-unital with unitisation $A^{\#}$, the canonical embedding $A \hookrightarrow S^{-1} A$ extends (canonically) to $A^{\#}$.

2. In general, if $A$ is a Banach algebra, $S^{-1} A$ - except under very restrictive conditions (see [13] and [14]) - is not a Banach algebra.

Let $D: A \rightarrow A$ be a derivation. Then $D$ has a unique extension, likewise denoted by $D$, to $S^{-1} A$ defined through

$$
D\left(\frac{a}{s}\right):=\frac{s D a-a D s}{s^{2}} \quad\left(\frac{a}{s} \in S^{-1} A\right) .
$$

Let $S_{0}$ be an arbitrary subset of $A$ containing no divisors of zero; we write $\left\langle S_{0}\right\rangle$ for the set of denominators generated by $S_{0}$. If $S_{0}=\left\{s_{1}, \ldots, s_{n}\right\}$, we also write $\left\langle s_{1}, \ldots, s_{n}\right\rangle$ instead of $\left\langle S_{0}\right\rangle$. Certainly, if $z \in A$ is such that $D z$ is not a divisor of zero, then $D z$ becomes invertible in $\langle D z\rangle^{-1} A$.

For our main theorem, we require sets of denominators with a particular property:

Definition 1.1: Let $A$ be a commutative algebra, and let $S \subset A$ be a set of denominators. Then $S$ is called ample if, for each $s_{1}, \ldots, s_{n} \in S$, there is $t \in S$ such that $t$ is divided by every element of $\left\langle s_{1}, \ldots, s_{n}\right\rangle$.

It is not hard to conclude from the Mittag-Leffler theorem that, in a commutative, topologically simple Banach algebra, the set of non-zero elements is an ample set of denominators. A very similar argument, however, shows that much smaller ample sets of denominators exist in abundance:

Proposition 1.2. Let $A$ be a commutative, topologically simple Banach algebra, and let $S_{0} \subset A$ be a countable set of non-zero elements. Then there is an ample, countable set of denominators $S \subset A$ containing $S_{0}$. 
Proof: Let $S_{0}=\left\{s_{n}: n \in \mathbb{N}\right\}$. We shall define inductively an increasing sequence. $\left(S_{n}\right)_{n=1}^{\infty}$ of countable sets of denominators in $A$ such that

(i) $s_{n} \in S_{n}$ for $n \in \mathbb{N}$, and

(ii) for each $n \in \mathbb{N}$, there is $t \in S_{n+1}$ such that $t$ is divided by every element of $S_{n}$.

By letting $S:=\bigcup_{n=1}^{\infty} S_{n}$, we obtain a set with the desired properties.

To start the induction, let $S_{1}:=\left\langle s_{1}\right\rangle$. Let $n \in \mathbb{N}$, and suppose that $S_{1}, \ldots, S_{n}$ have already been defined. Let $S_{n}=\left\{t_{n}: n \in \mathbb{N}\right\}$. Since $A$ is topologically simple, we have $\left(t_{n} A\right)^{-}=A$ for all $n \in \mathbb{N}$. From Bourbaki's Mittag-Leffler theorem (see, for example, [7]), it follows that $\bigcap_{n=1}^{\infty} t_{1} \cdots t_{n} A$ is also dense in $A$ and thus, in particular, non-zero. Let $t$ be an arbitrary, non-zero element of $\bigcap_{n=1}^{\infty} t_{1} \cdots t_{n} A$. Then, by definition, $t$ is divided by every element of $S_{n}$. Finally, let $S_{n+1}:=\left\langle S_{n} \cup\left\{s_{n+1}, t\right\}\right\rangle$.

\section{A POWER SERIES STRUCTURE THEOREM}

Let $A$ be a commutative, topologically simple Banach algebra, let $D: A \rightarrow A$ be a derivation, let $z \in A$ be such that $D z \neq 0$, and let $S \subset A$ be an ample set of denominators. Wish to prove an analogue of [15, Proposition 2.24], that is, a power series structure theorem for $S^{-1} A / \bigcap_{n=1}^{\infty} z^{n} S^{-1} A$. We follow [15] closely; whenever an argument is only a minor variation of one from [15], we just give a brief reference.

We begin with a purely algebraic lemma:

Lemma 2.1. Let $A$ be a commutative algebra with identity, let $\Delta: A \rightarrow A$ be a derivation, and let $z \in A$ be such that $\Delta z=1$. Define, for $n \in \mathbb{N}$,

$$
\theta_{n}(a):=\sum_{k=0}^{n}(-1)^{k} \frac{\Delta^{k} a}{k !} z^{k} \quad(a \in A)
$$

Then:

(i) $\theta_{n}(z)=0 \quad(n \in \mathbb{N})$;

(ii) $\Delta\left(\theta_{n}(a)\right) \in z^{n} A \quad(a \in A, n \in \mathbb{N})$;

(iii) $\theta_{n}(a b)-\theta_{n}(a) \theta_{n}(b) \in z^{n+1} A \quad(a, b \in A, n \in \mathbb{N})$;

(iv) $\theta_{n}\left(\theta_{m}(a)\right)-\theta_{m}(a) \in z^{m+1} A \quad(a \in A, n, m \in \mathbb{N}, m \geqslant n)$.

ProOF: The proof of [15, Lemma 2.15] is purely algebraic and thus carries over verbatim.

Let $A$ be a commutative Banach algebra, and let $z \in A$. Then $z$ is said to have finite closed descent if $\left(z^{n} A\right)^{-}=\left(z^{n+1} A\right)^{-}$for some $n \in \mathbb{N}[2]$. 
Lemma 2.2. Let $A$ be a commutative Banach algebra, let $z \in A$ have finite closed descent, let $S \subset A$ be an ample set of denominators, and let $s_{1}, \ldots, s_{N} \in S$. Then for any choice of sequences $\left(a_{k}\right)_{k=0}^{\infty}$ in $A$ and $\left(\nu_{k, 1}\right)_{k=0}^{\infty}, \ldots,\left(\nu_{k, N}\right)_{k=0}^{\infty}$ in $\mathbb{N}$, there is an element $y \in S^{-1} A$ and a sequence $\left(b_{n}\right)_{n=0}^{\infty}$ in $S^{-1} A$ such that

$$
\left(y-\sum_{k=0}^{n} \frac{a_{k}}{s_{1}^{\nu_{k, 1}} \cdots s_{N}^{\nu_{k}, N}} z^{k}\right)-z^{n+1} b_{n} \in \bigcap_{k=1}^{\infty} z^{k} S^{-1} A \quad\left(n \in \mathbb{N}_{0}\right)
$$

Proof: Since $S$ is ample, there is $t \in S$ such that $t$ is divided (in $A$ ) by every element of $\left\langle s_{1}, \ldots, s_{N}\right\rangle$; in particular, for each $k \in \mathbb{N}$, there is $t_{k} \in A$ such that

$$
t=t_{k} s_{1}^{\nu_{k, 1}} \cdots s_{N}^{\nu_{k, N}} \text {. }
$$

Define $\tilde{a}_{k}:=t_{k} a_{k}$ for $k \in \mathbb{N}$. Then the same Mittag-Leffler argument as in the proof of [15, Proposition 2.18] (which, in turn, goes back to [1]) yields $\tilde{y} \in A$ and a sequence $\left(\tilde{b}_{n}\right)_{n=0}^{\infty}$ in $A$ such that

$$
\left(\widetilde{y}-\sum_{k=0}^{n} \tilde{a}_{k} z^{k}\right)-z^{n+1} \widetilde{b}_{n} \in \bigcap_{k=1}^{\infty} z^{k} A \quad\left(n \in \mathbb{N}_{0}\right) .
$$

Division by $t$ (in $S^{-1} A$ ) yields the claim with $y:=\tilde{y} / t$ and $b_{n}:=\tilde{b}_{n} / t$ for $n \in \mathbb{N}$.

Let $A$ be any commutative algebra, and let $z \in A$ be such that $\bigcap_{n=1}^{\infty} z^{n} A=\{0\}$. The $z$-adic topology on $A$ is the metric topology defined through

$$
d(a, b):=\inf \left\{2^{-n}: n \in \mathbb{N}_{0} \text { and } a-b \in z^{n} A\right\} \quad(a, b \in A),
$$

where $z^{0} A:=A$. If $\bigcap_{n=1}^{\infty} z^{n} A \neq\{0\}$, we consider $A / \bigcap_{n=1}^{\infty} z^{n} A$. We write $\bar{a}$ for the coset of $a \in A$ in $A / \bigcap_{n=1}^{\infty} z^{n} A$. We then have a $\bar{z}$-adic topology on $A / \bigcap_{n=1}^{\infty} z^{n} A$.

In terms of the $\bar{z}$-adic topology, we obtain from Lemma 2.2 (compare [15, Proposition 2.18]):

Corollary 2.3. Let $A, z, S$ and $s_{1}, \ldots, s_{N}$ as well as $y$ and $b_{1}, b_{2}, \ldots$ be as in Lemma 2.2. Then the infinite series $\sum_{k=0}^{\infty}\left(a_{k} /\left(s_{1}^{\nu_{k, 1}} \cdots s_{N}^{\nu_{k, N}}\right)\right)^{-} \bar{z}^{k}$ converges to $\bar{y}$ and, for each $n \in \mathbb{N}_{0}$, the infinite series $\sum_{k=n+1}^{\infty}\left(a_{k} /\left(s_{1}^{\nu_{k, 1}} \cdots s_{N}^{\nu_{k, N}}\right)\right)^{-} \bar{z}^{k-n-1}$ converges to $\bar{b}_{n}$ in the $\bar{z}$-adic topology on $S^{-1} A / \bigcap_{k=1}^{\infty} z^{k} S^{-1} A$.

Corollary 2.4. Let $A$ be a commutative Banach algebra, let $z \in A$ have finite closed descent, let $D: A \rightarrow A$ be a derivation, and let $S \subset A$ be an ample set of 
denominators such that $D z$ is invertible in $S^{-1} A$. Then, for each $\bar{x} \in S^{-1} A / \bigcap_{n=1}^{\infty} z^{n} S^{-1} A$, the infinite series

$$
\theta(\bar{x}):=\sum_{k=0}^{\infty}(-1)^{k} \frac{\Delta^{k} \bar{x}}{k !} \bar{z}^{k}
$$

converges in $S^{-1} A / \bigcap_{n=1}^{\infty} z^{k} S^{-1} A$, where $\Delta$ is the derviation on $S^{-1} A / \bigcap_{n=1}^{\infty} z^{k} S^{-1} A$ induced by $(D z)^{-1} D$.

Proof: Slightly abusing notation, we also use $\Delta$ to denote the derivation $(D z)^{-1} D$ on $S^{-1} A$. Let $a \in A$ and $s \in S$ be arbitrary, and choose $b \in A$ and $t \in S$ such that $(D z)^{-1}=b / t$. We claim that, for each $k \in \mathbb{N}_{0}$, there are $c_{k} \in A$ and $\nu_{k}, \mu_{k} \in \mathbb{N}$ such that

$$
\Delta^{k}\left(\frac{a}{s}\right)=\frac{c_{k}}{s^{\nu_{k}} t^{\mu_{k}}}
$$

Since

$$
\Delta^{0}\left(\frac{a}{s}\right)=\frac{a}{s}=\frac{a t}{s t}
$$

the claim holds for $k=0$. Now suppose that the claim has been proved for arbitrary $k \in \mathbb{N}$. Then we have

$$
\Delta^{k+1}\left(\frac{a}{s}\right)=\Delta\left(\frac{c_{k}}{s^{\nu_{k}} t^{\mu_{k}}}\right)=\frac{b}{r} D\left(\frac{c_{k}}{s^{\nu_{k}} t^{\mu_{k}}}\right)=\frac{b\left(s^{\nu_{k}} t^{\mu_{k}} D c_{k}+c_{k} D\left(s^{\nu_{k}} t^{\mu_{k}}\right)\right)}{s^{2 \nu_{k}} t^{2 \mu_{k}+1}}
$$

which establishes the claim for $k+1$. The assertion of Corollary 2.4 then follows from Corollary 2.3 .

Invoking Lemma 2.1, we obtain as in the proof of [15, Lemma 2.20]:

Lemma 2.5. Let $A, z, D, S, \theta$ and $\Delta$ be as in Corollary 2.4. Then:

(i) $\theta(\bar{z})=\overline{0}$;

(ii) $\Delta \circ \theta=0$;

(iii) $\theta$ is a homomorphism.

(iv) $\theta$ is a projection onto a unital subalgebra $\mathcal{A}_{\theta}$ of $S^{-1} A / \bigcap_{n=1}^{\infty} z^{n} S^{-1} A$. Moreover, no non-zero element of $\mathcal{A}_{\theta}$ is divided by $\bar{z}$ in $S^{-1} A / \bigcap_{n=1}^{\infty} z^{n} S^{-1} A$.

As an analogue of $[\mathbf{1 5}$, Lemma 2.21], we obtain:

Proposition 2.6. Let $A, z, D, S, \Delta, \theta$ and $\mathcal{A}_{\theta}$ be as in Lemma 2.5, and suppose that $z$ is not a divisor of zero in $A$. Then, for each element $\bar{a} \in S^{-1} A / \bigcap_{n=1}^{\infty} z^{n} S^{-1} A$, there is a unique sequence $\left(\bar{a}_{k}\right)_{k=0}^{\infty}$ in $\mathcal{A}_{\theta}$ such that $\bar{a}=\sum_{k=0}^{\infty} \bar{a}_{k} \bar{z}^{k}$. Moroever, $\bar{z}^{m}$ with $m \in \mathbb{N}$ divides $\bar{a}$ in $S^{-1} A / \bigcap_{n=1}^{\infty} z^{n} S^{-1} A$ if and only if $\bar{a}_{0}=\cdots=\bar{a}_{m-1}=\overline{0}$. 
ProOF: We begin with a proof of the uniqueness of $\bar{a}_{0}, \bar{a}_{1}, \ldots$ Most of the corresponding arguments in the proof of [15, Lemma 2.21] carry over verbatim. All we need is a substitute for [15, Lemma 2.8(ii)]. To this end, let $x \in \bigcap_{n=1}^{\infty} z^{n} S^{-1} A$, and let $y \in S^{-1} A$ be such that $x=z y$. Furthermore, for any $\nu \in \mathbb{N}$, there is $\tilde{y} \in S^{-1} A$ such that $x=z^{\nu+1} \tilde{y}$. Since $z$ is not a divisor of zero in $A$, it cannot be a divisor of zero in $S^{-1} A$ either, that is, $y=z^{\nu} \widetilde{y}$. Since $\nu \in \mathbb{N}$ was arbitrary, this means that $y \in \bigcap_{n=1}^{\infty} z^{n} S^{-1} A$.

The existence of $\bar{a}_{0}, \bar{a}_{1}, \ldots$ is proved exactly as in the proof of [15, Lemma 2.21].

Finally, let $\bar{a}=\sum_{k=0}^{\infty} \bar{a}_{k} \bar{z}^{k} \in S^{-1} A / \bigcap_{n=1}^{\infty} z^{n} S^{-1} A$ with $\bar{a}_{0}, \bar{a}_{1}, \ldots \in \mathcal{A}_{\theta}$, and let $m \in \mathbb{N}$. As in the proof of [15, Lemma 2.21], we see that $\bar{a}_{0}=\cdots=\bar{a}_{m-1}=\overline{0}$ if $\bar{z}^{m}$ divides $\bar{a}$. Conversely, suppose that $\bar{a}_{0}=\cdots=\bar{a}_{m-1}=\overline{0}$. In the proof of the existence of $\bar{a}_{0}, \bar{a}_{1}, \ldots$, it is actually shown that there are $b_{0}, b_{1}, \ldots$ such that

$$
\bar{a}-\sum_{k=0}^{n} \bar{a}_{k} \bar{z}^{k}=\bar{z}^{n+1} \bar{b}_{n} \quad(n \in \mathbb{N}) .
$$

For $n=m-1$, this means $\bar{a}=\bar{z}^{m} \bar{b}_{m-1}$, that is, $\bar{z}^{m}$ divides $\bar{a}$.

As a consequence we obtain (compare [15, Lemma 2.22], whose proof takes over):

Corollary 2.7. Under the hypotheses of Proposition 2.6, we have the following:

(i) $\mathcal{A}_{\theta}=\operatorname{ker} \Delta$.

Moreover, if $\bar{a}=\sum_{k=0}^{\infty} \bar{a}_{k} \bar{z}^{k} \in S^{-1} A / \bigcap_{n=1}^{\infty} z^{n} S^{-1} A$ with $\bar{a}_{0}, \bar{a}_{1}, \ldots \in \mathcal{A}_{\theta}$, we have

(ii) $\Delta(\bar{a})=\sum_{k=1}^{\infty} k \bar{a}_{k} \bar{z}^{k-1}$;

(iii) $\bar{a}_{k}=\theta\left(\Delta^{k}(\bar{a})\right) / k$ ! for $k \in \mathbb{N}_{0}$.

For any commutative algebra $A$, let $A[[Z]]$ denote the algebra of formal power series with coefficients in $A$. The formal derivative is the derivation

$$
A[[Z]] \rightarrow A[[Z]], \quad \sum_{k=0}^{\infty} a_{k} Z^{k} \mapsto \sum_{k=1}^{\infty} k a_{k} Z^{k-1} .
$$

We now have all the ingredients ready for the proof of the following structure result:

Theorem 2.8. Under the hypotheses of Proposition 2.6, the map

$$
S^{-1} A / \bigcap_{n=1}^{\infty} z^{n} S^{-1} A \rightarrow \mathcal{A}_{\theta}[[Z]], \quad \bar{a} \mapsto \sum_{k=0}^{\infty} \frac{\theta\left(\Delta^{k} \bar{a}\right)}{k !} Z^{k}
$$

is an algebra monomorphism, whose range contains the polynomials with coefficients in $\mathcal{A}_{\theta}$. Morover, $\Delta$ acts on the range of $(*)$ as the restriction of the formal derivative. 
PROOF: As noted in the proof of the corresponding assertion in [15] (Proposition 2.24), most of Theorem 2.8 has already been established (Lemma 2.5, Proposition 2.6, and Corollary 2.7).

What remains to be shown is the multiplicativity of $(*)$. Here, the corresponding argument from [15] requires some modification. Let $\bar{a}, \bar{b} \in S^{-1} A / \bigcap_{n=1}^{\infty} z^{n} S^{-1} A$ be such that $\bar{a}=\sum_{k=1}^{n} \bar{a}_{k} \bar{z}^{k}$ and $\bar{b}=\sum_{k=1}^{n} \bar{b}_{k} \bar{z}^{k}$ with $\bar{a}_{0}, \bar{b}_{0}, \ldots, \bar{a}_{n}, \bar{b}_{n} \in \mathcal{A}_{\theta}$. Then, as in the proof of $[\mathbf{1 5}$, Proposition 2.24], we see that (*) maps $\bar{a} \bar{b}$ to the product of the images of $\bar{a}$ and $\bar{b}$ under (*). Since $(*)$ is continuous with respect to the $\bar{z}$-adic topology on $S^{-1} A / \bigcap_{n=1}^{\infty} z^{n} S^{-1} A$ and the $Z$-adic topology on $\mathcal{A}_{\theta}[[Z]]$, it follows that $(*)$ is indeed multiplicative.

Note that, unlike in [15, Proposition 2.24], we cannot conclude that $(*)$ is onto: This is due to the restriction on the denominators we had to impose in Lemma 2.2.

In a commutative, topologically simple Banach algebra, every non-zero element is not a divisor of zero and has finite closed descent. Hence, we have:

Corollary 2.9. Let $A$ be a commutative, topologically simple Banach algebra, let $S \subset A$ be an ample set of denominators, let $D: A \rightarrow A$ be a derivation, and let $z \in A$ be such that $D z$ is invertible in $S^{-1} A$. Then, with $\Delta$ denoting the derivation on $S^{-1} A / \bigcap_{n=1}^{\infty} z^{n} S^{-1} A$ induced by $(D z)^{-1} D$ and with

$$
\theta: S^{-1} A / \bigcap_{n=1}^{\infty} z^{n} S^{-1} A \rightarrow S^{-1} A / \bigcap_{n=1}^{\infty} z^{n} S^{-1} A, \quad \bar{x} \mapsto \sum_{k=0}^{\infty}(-1)^{k} \frac{\Delta^{k} \bar{x}}{k !} \bar{z}^{k},
$$

the map $(*)$ is an algebra monomorphism, whose range contains the polynomials with coefficients in $\mathcal{A}_{\theta}$. Morover, $\Delta$ acts on the range of $(*)$ as the restriction of the formal derivative.

REMARKS 1. Let $A$ be any commutative, topologically simple Banach algebra, let $D$ : $A \rightarrow A$ be a derivation, and let $z \in A$ be such that $D z \neq 0$. By Proposition 1.2, the element $D z$ is then contained in a countable, ample set $S \subset A$ of denominators. Then certainly $D z$ is invertible in $S^{-1} A$, so that Corollary 2.9 applies. However, we do not know that $z$ is not invertible in $S^{-1} A$. If $z$ is invertible, then both $S^{-1} A / \bigcap_{n=1}^{\infty} z^{n} S^{-1} A$ and $\mathcal{A}_{\theta}$ are just the zero algebra. In this situation, the conclusion of Corollary $2.9-$ albeit true - is not very interesting.

2. The most interesting consequence of [15, Proposition 2.24] is certainly [15, Theorem 2.25], which eventually leads to a contradiction and thus to a proof of the SingerWermer conjecture. It is not hard to see that, in our setting, we have an analogue of [15, Proposition 2.24]. In [15], Thomas establishes a contradiction to [15, Theorem 2.25] through the construction of recalcitrant systems $[15$, Definition 3.3]. We have been unable 
so far to construct (analogues of) recalcitrant systems in localisations of commutative, topologically simple Banach algebras.

\section{REFERENCES}

[1] G. R. Allan, 'Embedding the algebra of formal power series in a Banach algebra', Proc. London Math. Soc. (3) 25 (1972), 329-340.

[2] G.R. Allan Elements of finite closed descent in a Banach algebra, J. London Math. Soc. (2) 7 (1973), 462-466.

[3] M.F. Atiyah and I. G. MacDonald, Introduction to commutative algebra (Addison-Wesley, Massachusetts, London, Ontario, 1969).

[4] P.C. Curtis, Jr., 'Derivations in commutative Banach algebras', in Radical Banach algebras and automatic continuity, (J.M. Bachar et al., Editors), Springer Lecture Notes in Mathematics 975 (Springer Verlag, Berlin, Heidelberg, New York, 1983), pp. 328-333.

[5] J. Cusack, 'Automatic continuity and topologically simple radical Banach algebras', $J$. London Math. Soc. (2) 16 (1977), 493-500.

[6] P.G. Dixon, 'Semiprime Banach algebras', J. London Math. Soc. (2) 6 (1973), 676-678.

[7] J. Esterle, 'Mittag-Leffler methods in the theory of Banach algebras and a new approach to Michael's problem', in Proceedings of the Conference on Banach Algebras and Several Complex Variables, (F. Greenleaf and D. Gulick, Editors), Contemporary Mathematics 32 (Amer. Math. Soc., Providence R.I., 1984), pp. 107-129.

[8] R.V. Garimella, 'Continuity of derivations on some semiprime Banach algebras', Proc. Amer. Math. Soc. 99 (1987), 289-292.

[9] E. Illoussamen, 'Continuité des dérivations et des épimorphismes dans certaines algèbres de Banach', Rend. Circ. Mat. Palermo (2) 44 (1995), 173-186.

[10] B.E. Johnson, 'Continuity of derivations on commutative algebras', Amer. J. Math. 91 (1969), 1-10.

[11] V. Runde, 'Automatic continuity of derivations and epimorphisms', Pacific J. Math. 147 (1991), 365-374.

[12] I.M. Singer and J. Wermer, 'Derivations on commutative normed algebras', Math. Ann. 129 (1955), 260-264.

[13] I. Suciu, 'Eine natürliche Erweiterung der kommutativen Banachalgebren', Rev. Roum. Math. Pures Appl. 7 (1962), 483-491.

[14] I. Suciu, 'Bruchalgebren der Banachalgebren', Rev. Roum. Math. Pures Appl. 8 (1963), 313-316.

[15] M.P. Thomas, 'The image of a derivation is contained in the radical', Ann. of Math. 128 (1988), 435-460.

Ecole Normale Supérieure Takaddoum

Département de Mathématiques

B.P. 5118

10105 Rabat

Morocco
Fachbereich 9 Mathematik

Universität des Saarlandes

Postfach 151150

66041 Saarbrücken

Germany

e-mail: runde@math.uni-sb.de 